Reprod. Nutr. Dévelop., 1980, 20 (2), 377-379.

\title{
Préface
}

\section{Biologie du développement et santé de l'enfant}

par P. ROYER

Conseiller pour la biologie ef la médecine.

DGRST - 35, rue St Dominique, 75007 Paris.

L'action concertée « Biologie de la Reproduction et du Développement 》 a bientôt dix ans d'âge. Son succès est réel et les articles qui suivent illustrent bien certaines des acquisitions qui lui sont dues. Ayant eu l'honneur de présider son Comité pendant 7 ans, je voudrais commenter l'importance de certains de ces résultats dans un secteur qui m'est familier : la protection de la Santé de l'Enfant.

On connaît la baisse de fécondité et la diminution de la natalité observées dans la plupart des pays industriels depuis plusieurs années, y compris en France. L'INSEE vient de publier son estimation du nombre de naissances dans notre pays en 1978 , à savoir 735000 . Dans le même temps on assiste à une baisse impressionnante du taux de mortalité infantile définie par les morts-nés et les décès jusqu'à l'âge d'un an. Ce taux était de 100 pour 1000 en 1946, 20 pour 1000 en 1968. Depuis cette date il n'a cessé de décroître de façon régulière : 14,6 en 1974, 13,6 en 1975, 12,6 en 1976, 11,5 en 1977 ef 10,6 en 1978 - ce qui place notre pays près de la Suède, du Danemark et des Pays-Bas, au voisinage de 10 p. 1000 , taux le plus faible atteint dans le monde. Cette amélioration tient à une très forte action dans le domaine de la recherche coordonnée à des décisions heureuses en matière d'organisation sociale, hospitalière et universitaire. Cette amélioration porte également sur le taux de mortalité périnatale qui mesure les progrès en obstétrique et en médecine du nouveau-né. Cette baisse de la natalité et celle de la mortalité périnatale et infantile ne se compensent pas par malheur. Elles se situent dans un cadre plus large de type sociétal. Les jeunes couples veulent plus que jadis maîtriser leur procréation, décider des dates de conception, choisir le nombre, le sexe, la qualité biologique des enfants à venir. Ceci reste en partie un simple espoir. Mais il est à peu près certain qu'on y répondra dans les années à venir.

La biologie du développement est une discipline aujourd'hui en pleine vigueur dans notre pays. Ses découvertes s'appliquent de façon immédiate au grand secteur de la santé de l'enfant. Les stérilités et les échecs de la reproduction vont connaître de nouvelles solutions : le développement récent ef brillant de l'insémination artificielle dans les stérilités masculines actuelles ou potentielles; le traitement des stérilités tubaires par microchirurgie et aussi le développement des réimplantations utérines des ovocyles fécondés in vitro est à souligner. La protection du fœetus et du nou- 
veau-né contre la naissance prématurée ef contre la malnutrition devrait très vite aboutir à des résultats extraordinaires. Le taux de la prématuration, 11 p. 100 des naissances en 1970, afteint maintenant 5 à 6 p. 100 en France et on peut espérer voir baisser ce chiffre. Ceci laisse entendre la chute du taux des handicaps moteurs, mentaux et respiratoires dûs à la naissance prématurée. La solution partielle du problème des handicaps dont la prise en charge totale représenterait 2 p. 100 de la production intérieure brute (PIB), plus que tout le financement de la recherche scientifique et technique en France, serait une application chargée d'une valeur ajoutée humaine extraordinaire. Reste le problème accablant des malformations congénitales. Trois pour cent des enfants sont atteints d'une malformation congénitale plus ou moins grande. Environ le tiers est dû à une agression pendant la grossesse par un virus, un toxique, des rayonnements ionisants. Un tiers dépend d'une maladie héréditaire. Un tiers est lié à une aberration chromosomique. S'en rapprochent les maladies héréditaires du métabolisme ou de la défense immunitaire. Ceci pour les causes connues, beaucoup de malformations restant mystérieuses. Ces malformations sont responsables de 15 p. 100 des morts fœetales tardives, de 18 p. 100 des morts pendant les premiers mois de la vie. Elles affectent surtout les membres, le système nerveux, les appareils digestif, urinaire et cardiovasculaire. Elles sont, après les accidents, la seconde cause de mortalité durant la petite enfance dans les pays favorisés où elles représentent la cause principale de l'hospitalisation lourde et des handicaps de l'enfant. La charge sociale, affective, médicale et financière des survivants est énorme. Des progrès récents ont certes déjà permis de prévenir parfois - comme pour la rubéole, la thalidomide, les radiations ionisantes - et de détecter dès les premiers mois de la gestation de nombreuses malformations du système nerveux, des membres ef des reins ef des maladies héréditaires du métabolisme grâce à l'échographie et à l'examen du liquide amniotique et de ses cellules. Il est clair qu'un effort privilégié doit se maintenir dans le futur pour essayer de mieux comprendre, prévenir et détecter, traiter ces malformations. Le bénéfice pour la Société, exprimé en termes de démographie, d'économie et d'affectivité, serait optimal.

Avancer dans ce domaine signifie progresser dans la connaissance du programme et des erreurs du développement de l'embryon. Or, c'est là l'un des problèmes les plus difficiles de la biologie. Longtemps restée descriptive, cette étude utilise depuis quelques années les méthodes de la biologie moléculaire chaque fois qu'elle le peut. Les connaissances sur les mécanismes qui sous-tendent le développement de l'embryon restent encore limitées. On sait que ce développement correspond à l'exécution du programme génétique inscrit, sous forme d'instructions codées, dans les chromosomes de l'œuf fécondé. Ces instructions contiennent les plans d'architecte du futur organisme et de ses constifuants ainsi que les moyens de mettre ces plans à exécution dans le temps et dans l'espace. Chaque cellule contient dans son noyau tous les gènes de l'organisme. Cependant, à mesure que se développe l'embryon, les cellules se multiplient, changent d'activités chimiques, se déplacent, réagissent avec d'autres cellules de manière ordonnée. Ainsi se mettent en place les formes, les organes, les tissus, les fonctions intégrées. Pour accomplir chacune de ces transformations graduelles, les cellules n'utilisent qu'une fraction de leurs gènes. L'activation de certains gènes à des moments précis du développement ef les changements cellulaires qui en résultent sont déclenchés par l'embryon. Certains gènes sont actifs une fois seulement et pen- 
dant un temps limité durant le cycle de vie de l'organisme. Bien des anomalies congénitales et bien des maladies qui surviendront plus tard dans la vie sont dues à des erreurs dans cet ordonnancement. De sa connaissance partielle peuvent naître une connaissance meilleure, une détection, une prévention.

Ainsi, il faut souhaiter que cette tâche vigoureuse d'incitation et de coordination de la recherche qui a été permise par l'action concertée se poursuive à travers des programmes nouveaux élaborés par les universités, par les organismes de recherche - CNRS, INSERM et INRA - ef par la DGRST. 